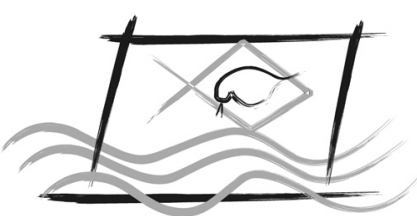

ECOTOX - BRASIL

\title{
Análise de risco ecológico com o uso do Bioindicador Lolium multiflorum
}

\author{
M.S. Rodrigues ${ }^{1 *}$, M.T.M Raya-RodrigueZ ${ }^{2}$ \\ ${ }^{1}$ Instituto Federal de Educação, Ciência e Tecnologia do Rio Grande do Sul, Porto Alegre, RS, Brasil. \\ ${ }^{2}$ PPG em Ecologia, Universidade Federal do Rio Grande do Sul, Porto Alegre, RS, Brasil. \\ ${ }^{*}$ Corresponding author: Magali da Silva Rodrigues, Rua Coronel Vicente, 281 - Centro Histórico- POA/RS - CEP: 90.030-041 \\ $3^{\circ}$ andar- sala 325 - Porto Alegre- RS- Brasil.
}

(Received January 7, 2009; Accept December 30, 2010)

\begin{abstract}
Resumo
Este trabalho foi desenvolvido no período de janeiro de 2005 a março de 2008, totalizando 39 meses de estudo. Foi realizado no

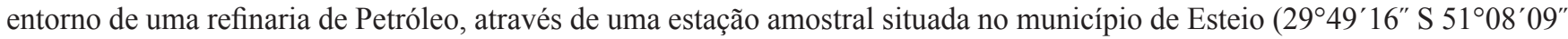
O), Rio Grande do Sul, Brasil. O objetivo desse trabalho foi desenvolver e aplicar um modelo de Análise de Risco Ecológico (ARE) com vistas a classificar os riscos associados às emissões atmosféricas em áreas industriais a partir da utilização do bioindicador vegetal Lolium multiflorum. O modelo proposto associou as informações provenientes do efeito dos estressores, oriundos das emissões atmosféricas de compostos de enxofre, nos receptores, ou seja, o tecido do bioindicador vegetal. Foi avaliado nos bioindicadores o percentual de enxofre acumulado classificado, neste estudo, como variável resposta. Esta foi integrada aos dados de concentração de $\mathrm{SO}_{2}$ da atmosfera, que deram origem as variáveis explicativas, unidade de potencial tóxico (UPT) e unidade tóxica de exposição (UTE). Esta integração foi realizada a partir da análise estatística multivariada de correspondência que demonstrou indícios de risco à vegetação do entorno da refinaria quando estas foram submetidas a UPT maiores do que a unidade.
\end{abstract}

Palavras-chave: avaliação de risco ecológico, biomonitoramento, enxofre, Lolium multiflorum, poluição atmosférica.

\section{Ecological risk analysis using the vegetable Bioindicator Lolium multiflorum}

\begin{abstract}
This work was starting from January, 2005 to March, 2008, during 39 months of monitoring. This evaluation was developed around of Petroleum Refinery area, in one fixed sample station localized in Esteio (2949'16" S 51 00 '09') cities, Rio Grande do Sul state, Brazil. The objective of this work was to develop and apply a model Ecological Risk Analysis (ERA) to classify the risks related to the atmospheric emissions in industrial areas using the vegetable bioindicator Lolium multiflorum. The proposed model for the ecologic risk evaluation associates the information from stressors' effects, which come from sulfur compounded gases emissions, at the receptors, that is, the vegetable bioindicator tissue. It was evaluated in the vegetable bioindicators parameter; sulfur concentration accumulated in the plants, classified in the study as "answers data". These were linked to the $\mathrm{SO}_{2}$ atmosphere concentration, which generate the "consequence data"; Toxic Potential Unit (TPU) and Exposure Toxic Unit (ETU). The integration was made using the statistics correspondence multiple variable analysis that demonstrate indications of risks for the vegetation localized around the refinery, when it was exposed an TPU higher than the unit.
\end{abstract}

Key words: atmospheric pollution, biomonitoring, Lolium multiflorum, risk assessment, sulphur.

*Corresponding author: Magali Rodrigues, e-mail: magali.rodrigues@poa.ifrs.edu.br 


\section{INTRODUÇÃO}

Estudos recentes apontam a Análise de Risco Ecológico (ARE) como uma importante ferramenta para avaliar a sustentabilidade dos ecossistemas, além de ser um instrumento fundamental dos processos de decisão sobre a gestão de locais poluídos, permitindo avaliação mais precisa dos riscos reais para os receptores ecológicos potencialmente afetados pelo(s) contaminante(s) (Junger, et al., 2005, Sousa, 2005; Nakagome, et al., 2006; Arias, et al., 2007; Niemeyer, 2007, Froehmer \& Martins, 2008). A ARE é uma metodologia científica para quantificar ou perceber os riscos do efeito adverso de um contaminante na saúde humana ou ao meio ambiente (Mines \& Lackey, 2009). Muito embora a ARE seja fortemente influenciada pela comparação das concentrações ambientais com os valores orientadores, qualquer esquema de ARE deve possuir uma forte componente de bioensaios, tais como o uso de testes toxicológicos, biomarcadores ou bioindicadores (Parkhurst, 1996, Souza, 2005, Niemeyer, 2007). Estes são fundamentais em todo o processo, pois incorporam a interação entre os contaminantes e as propriedades ambientais, possibilitando avaliação ecologicamente relevante dos efeitos derivados da exposição.

No Brasil a ARE ainda não é utilizada como ferramenta para cumprimento das leis ambientais ou como norteadora das tomadas de decisões de proteção ambiental (Pedrozo, et al., 2002). As etapas no processo de avaliação de risco ecológico são representadas pelas seguintes atividades (USEPA, 1997): formulação do problema, caracterização da exposição ambiental (screening), caracterização dos efeitos ecológicos, caracterização do risco e critérios de decisão. Cabe ressaltar que a aplicação de tal ferramenta se faz notória em atividades industriais que provocam a emissão de gases para a atmosfera, devido principalmente ao potencial de sinergismo desses gases no ambiente.

Tendo em vista que a gramínea $L$. multiflorum é um bioindicador de acumulação que apresenta tolerância frente à poluição atmosférica, esta planta é bastante utilizada para avaliar a poluição em locais de grande concentração de enxofre (Divan, 1994). Estudos apontam que há redução de crescimento de L. multiflorum quando estes são submetidos a concentrações elevadas de $\mathrm{SO}_{2}$ (Crittenden \& Read, 1979, Ashenden \& Willians, 1980). Neste sentido, é possível avaliar os riscos ecológicos provenientes de emissões atmosféricas a partir do efeito dos estressores (compostos de enxofre) sobre os organismos (L. multiflorum), através da concentração de enxofre acumulado na parte aérea das folhas. Desta forma, este trabalho teve por objetivo desenvolver e aplicar um modelo de Análise de Risco Ecológico (ARE) com vistas a classificar os riscos associados às emissões atmosféricas em áreas industriais a partir da utilização do bioindicador vegetal Lolium multiflorum e da aplicação de um modelo semelhante ao utilizado para análise de risco em ambientes aquáticos (Parkhurst, 1996).

\section{MATERIAL E MÉTODOS}

Plantas de L. multiflorum foram cultivadas em ambiente isento de contaminação localizado no Centro de Ecologia/ UFRGS. Um sistema de vasos contendo solo adubado foi utilizado sobre reservatórios com água. Após o período de cultivo, três vasos da espécie foram expostos por aproximadamente 30 dias na estação amostral de Esteio, RS. A estação amostral foi escolhida para o desenvolvimento do trabalho por apresentar os dois tipos de monitoramento o químico e o biológico. O desenvolvimento do trabalho englobou quatro etapas: formulação do problema, avaliação da concentração de compostos gasosos de enxofre no entorno da estação amostral, avaliação dos bioindicadores vegetais e aplicação de modelo de análise de risco ecológico (ARE).

$\mathrm{Na}$ primeira etapa foram identificados os principais contaminantes e as principais fontes de contaminação. Os dados de $\mathrm{SO}_{2}$ utilizados foram os oriundos da rede automática de monitoramento químico, os quais são continuamente analisados pelo equipamento Environnement, fornecendo resultados de concentrações $\left(\mu \mathrm{g} \cdot \mathrm{m}^{-3}\right)$ a cada 15 minutos. O princípio químico de medição deste poluente está baseado no método de Fluorescência Ultravioleta com faixa de medição de 0 a $1 \mathrm{ppm}\left(1 \times 10^{3} \mu \mathrm{g} \cdot \mathrm{m}^{-3}\right)$ e limite de detecção de $2,62 \mu \mathrm{g} \cdot \mathrm{m}^{-3}$.

A segunda etapa contou com a avaliação dos níveis de contaminação de $\mathrm{SO}_{2}$ medidos a partir do monitoramento químico, estes foram comparados com o padrão primário $\left(80 \mu \mathrm{g} . \mathrm{m}^{-3}\right)$ e secundário $\left(40 \mu \mathrm{g} . \mathrm{m}^{-3}\right)$, valores de referência nacional (Resolução CONAMA 03/90). Os dados de concentração de $\mathrm{SO}_{2}$ foram tratados de forma que os períodos do monitoramento químico coincidissem com o período de exposição dos bioindicadores vegetais. $\mathrm{O}$ padrão secundário de $\mathrm{SO}_{2}$, foi comparado com estudos realizados com $L$. multiflorum que indicaram redução de crescimento quando estes foram submetidas a concentrações de $50 \mu \mathrm{g} . \mathrm{m}^{-3} \mathrm{SO}_{2}$ (Crittenden \& Read, 1979). A partir dos conceitos existentes utilizados para avaliação de risco ecológico em ambientes aquáticos, quais sejam: "Unidade Tóxica" (Tarazona, 2000) e "Quociente de Risco" (USEPA, 1989) foram estabelecidas, neste modelo, as seguintes definições:

a) Potencial Tóxico Agudo $\Rightarrow$ sempre que ocorrerem concentrações ambientais de $\mathrm{SO}_{2}$ superiores ao padrão primário da qualidade do ar. São consideradas as médias dos picos de concentração tóxica aguda para o período de exposição.

b) Potencial Tóxico Crônico $\Rightarrow$ sempre que ocorrerem concentrações ambientais de $\mathrm{SO}_{2}$ superiores ao padrão secundário da qualidade do ar. São consideradas as médias dos picos de concentração tóxica crônica para o período de exposição.

c) Unidade Potencial Tóxica (UPT) ou Unidade Tóxica (UT) $\Rightarrow$ é o quociente entre a concentração ambiental média de $\mathrm{SO}_{2}$ que ultrapassou o padrão primário de qualidade do ar (concentração pico) e o padrão secundário da qualidade do ar. O padrão secundário da qualidade do ar foi utilizado, pois está na mesma ordem de grandeza da concentração testada 
no estudo dos autores Crittenden \& Read, 1979 Este índice indica as concentrações ambientais de $\mathrm{SO}_{2}$ que podem afetar o crescimento das plantas bioindicadoras através de efeito crônico.

d) Unidade Tóxica de Exposição (UTE) ou Potencial Tóxico de Exposição (PTE) $\Rightarrow$ é o produto da UPT e o período de exposição dos bioindicadores à concentração superior ao padrão primário da qualidade do ar. Este índice indica as concentrações ambientais de $\mathrm{SO}_{2}$ que podem afetar às plantas bioindicadoras através de efeito agudo. Foi considerado como indício de risco a seguinte classificação (Tarazona, 2000):

$\mathrm{UPT} \leq 1 \Rightarrow$ indica risco ecológico pouco provável, neste caso, o risco poderá ser considerado desprezível.

UPT $>1 \Rightarrow$ considera-se que há possibilidade de potenciais efeitos adversos ao sistema ecológico.

Na terceira etapa foirealizada a avaliação dos bioindicadores a partir dos ensaios de acumulação de enxofre (LECO, 1994). Para o preparo das amostras foram utilizadas em torno de $300 \mathrm{mg}$ de folhas de L. multiflorum, previamente secas em estufa a $80^{\circ} \mathrm{C}$ por 7 dias e moídas em moinho de facas com malha de 30 mesh. O percentual de enxofre foi determinado pela análise instrumental com limite de detecção analítica de $0,001 \%$ através princípio da absorção de energia no espectro do infravermelho. Na etapa final, os resultados obtidos com a avaliação química foram integrados com os resultados dos ensaios biológicos através do uso de análise estatística multivariada de correspondência, com vistas à determinação dos indícios de risco (Souza, 2005; Niemeyer, 2007).

\section{RESULTADOS}

A partir dos dados de qualidade ambiental existentes em referências bibliográficas "guidelines" (Crittenden \& Read, 1979; Resolução CONAMA 03/90; USEPA, 1989, Tarazona, 2000, Klumpp, 2001) e dos histogramas de freqüência foram estabelecidos os pontos de corte para estimar as classes de risco ecológico para os parâmetros avaliados, citam-se o $\mathrm{SO}_{2}$ médio da atmosfera (Figura 1), Unidade Potencial Tóxica (UPT) (Figura 2) e Unidade Tóxica de Exposição (UTE) (Figura 3) consideradas, neste modelo, como variáveis explicativas, além da variável resposta, enxofre acumulado na parte aérea das plantas (Figura 4). Pela análise da Figura 1, observa-se que os indícios de risco para a concentração de $\mathrm{SO}_{2}$ médio na atmosfera se mostraram predominantemente na classe 1 , nível baixo, ou seja, concentrações inferiores ao padrão secundário da qualidade do ar, as quais se prevê o mínimo efeito adverso sobre o bem-estar da população, assim como o mínimo dano à fauna, à flora, aos materiais e ao meio ambiente em geral (Resolução CONAMA 03/90). A distribuição entre as categorias foi de $79 \%$ de indício de risco baixo, $18 \%$ médio e $3 \%$ alto. A média e o desvio-padrão da variável explicativa, $\mathrm{SO}_{2}$, para o período foi de $27 \pm 22 \mu \mathrm{g} . \mathrm{m}^{-3}$.

A classificação dos indícios de risco da Unidade Potencial Tóxica (UPT) teve por base os conceitos de Unidade Tóxica (Tarazona, 2000) e Quociente de Risco (USEPA, 1989). Estes se situaram predominantemente na classe 2 , nível médio de risco em $67 \%$ do período de monitoramento, seguido de $21 \%$ de risco baixo e $13 \%$ de alto (Figura 2). A média e o desviopadrão da variável explicativa UPT para o período de estudo, foi de $3,0 \pm 1,1$. A Unidade Tóxica de Exposição (UTE) foi determinada a partir do produto da Unidade Potencial Tóxica (UPT) com o tempo de exposição do bioindicador a concentração pico, ou seja, concentração de $\mathrm{SO}_{2}$ superior ao padrão primário de qualidade do ar, $80 \mu \mathrm{g} . \mathrm{m}^{-3}$ (Resolução CONAMA 03/90). Para este estudo definiu-se que as plantas do entorno da Refinaria sofreriam supostamente algum efeito com indício de risco alto quando submetidas a períodos de exposição de pico superiores a 20 horas (Crittenden \& Read, 1979). Os indícios de risco para a UTE apresentaram classificação de risco alto para $56 \%$ dos valores medidos, seguidos de $41 \%$ de risco baixo e 3\% de médio (Figura 3)

Para a variável resposta, teor de enxofre acumulado na parte área do bioindicador L. multiflorum, a determinação dos intervalos de corte para classificação do risco foi baseada nas classes de valores-limites deste composto, propostas por Klumpp et al.(2001). No total de meses avaliados no estudo, 39 meses, obteve-se $46 \%$ como de nível médio, 38\% nível alto e $15 \%$ classificado como de nível baixo, ou seja, tiveram valores inferiores a $0,292 \%$ (em peso seco). A média e o desvio-padrão da variável explicativa enxofre foliar foi de $0,519 \pm 0,276 \%$

A categorização das classes de risco, estabelecida para as variáveis explicativas teor de $\mathrm{SO}_{2}$ no ar, UPT, UTE e a variável resposta, teor de enxofre acumulado na parte aérea das plantas (enxofre foliar), permitiu que estas fossem integradas a partir da análise estatística multivariada de correspondência através do uso do software SPSS, versão 13.0 for Windows (Figura 5). A integração destas proporcionou o agrupamento em três diferentes classes de risco, quais sejam: grupo com indício de risco baixo, médio e alto. A formação de grupos distintos, a partir da integração destas variáveis, é a chave que comprova a validação do modelo proposto o qual visa avaliar os efeitos danosos da poluição atmosférica por compostos gasosos de enxofre sobre a vegetação do entorno de localidades industriais (Souza, 2005; Niemeyer, 2007).

\section{DISCUSSÃO}

A partir da comparação do histograma de freqüência para o teor de $\mathrm{SO}_{2}$ da atmosfera (Figura 1) com o histograma da Unidade Potencial Tóxica (UPT) (Figura 2) verifica-se que embora a concentração média de $\mathrm{SO}_{2}$ na atmosfera para o período amostral tenha oscilado entre as classes 1 e 2, ou seja, de risco baixo a médio, a variável UPT apresentou maiores indícios de risco. Esta diferença justifica-se, pois os dados do teor de $\mathrm{SO}_{2}$ na atmosfera são representados por valores médios dos períodos amostrais, já para a variável UPT os valores considerados foram os valores pico, ou seja, valores superiores ao padrão primário da qualidade do ar, $80 \mu \mathrm{g} \cdot \mathrm{m}^{-3}$ (Resolução CONAMA 03/90). A partir desta avaliação, cabe ressaltar que embora a concentração média de $\mathrm{SO}_{2}$ tenha sido 


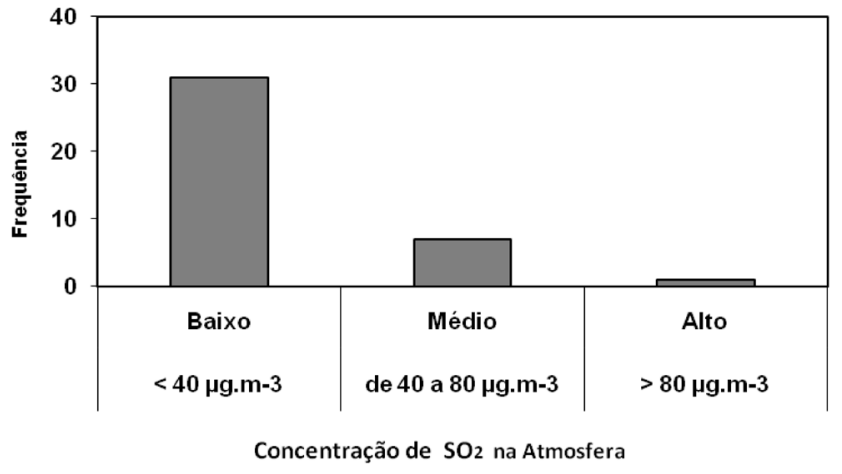

Figura 1 - Histograma de frequências para a classificação dos indícios de risco da variável teor de $\mathrm{SO}_{2}$ médio da atmosfera na estação amostral de Esteio, RS.

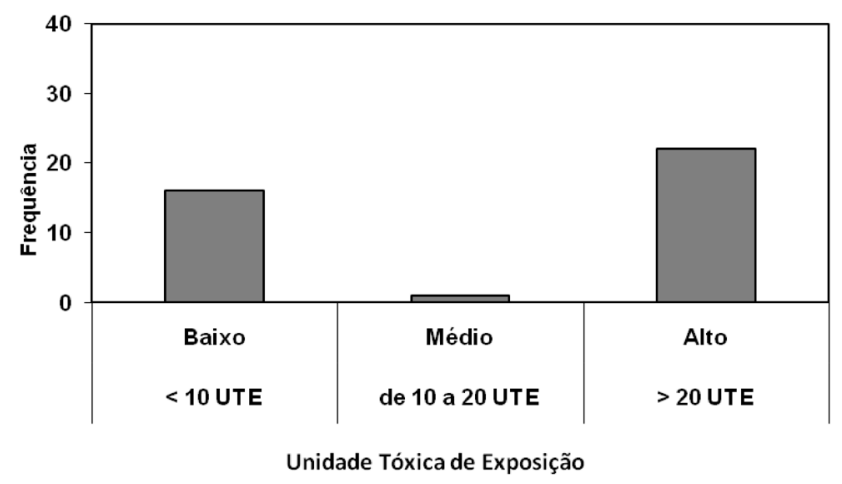

Figura 3 - Histograma de frequências para a classificação dos indícios de risco da variável Unidade Tóxica de Exposição (UTE) ou PTE (Potencial

Tóxico de Exposição) na estação amostral de Esteio, RS.

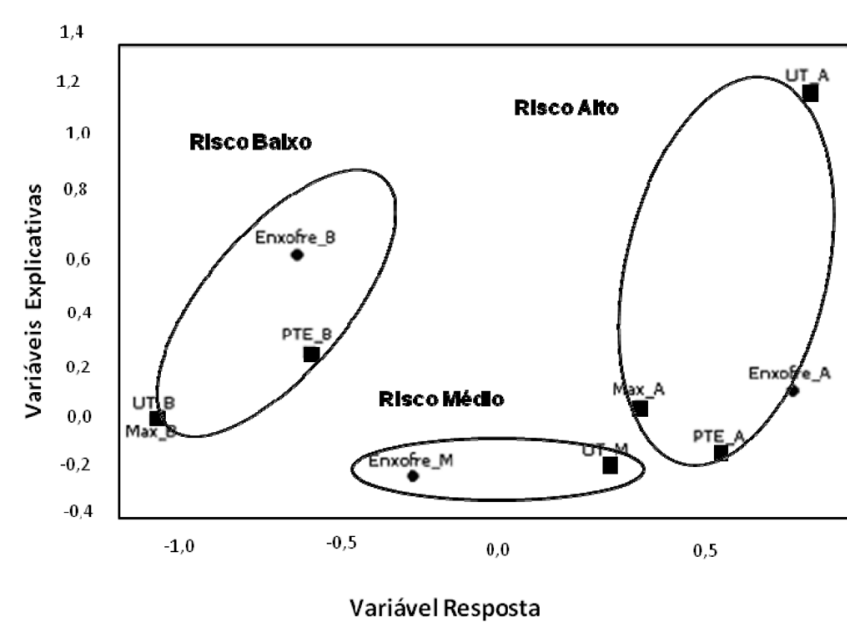

Figura 5 - Representação gráfica da integração da variável resposta, enxofre foliar acumulado na parte aérea da gramínea padronizada de L. multiflorum com o máximo valor de $\mathrm{SO}_{2}$ atmosférico observado no período e as variáveis explicativas, PTE e UT na estação amostral de Esteio, RS

mantida dentro de níveis de risco aceitáveis para as classes 1 e 2 (Figura 1), as plantas do entorno da Refinaria estão sujeitas à concentração pico e o efeito sobre a vegetação pode ser medido a partir da variável resposta de teor de enxofre acumulado na parte aérea das plantas (Figura 4).

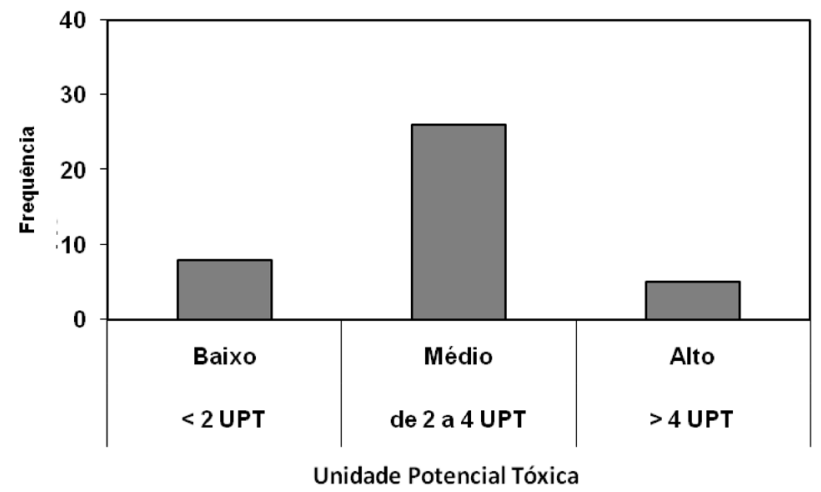

Figura 2 - Histograma de frequências para a classificação dos indícios de risco da variável Unidade Potencial Tóxica (UPT) ou Unidade Tóxica (UT) na estação amostral de Esteio, RS.

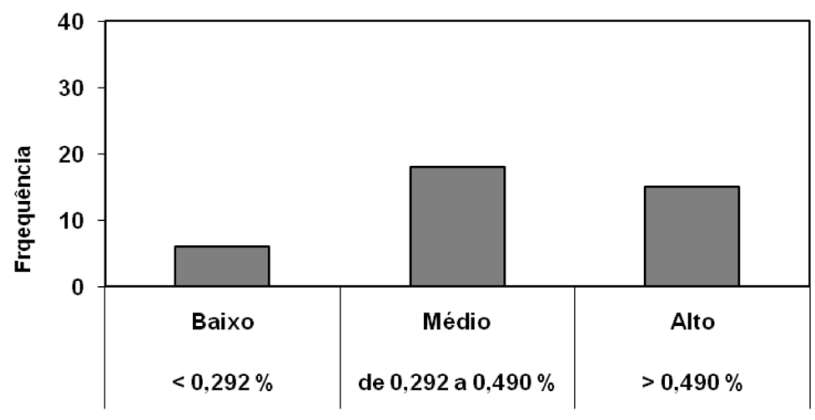

Teor de enxofre acumulado na parte área das plantas

Figura 4 - Histograma de frequências para a classificação dos indícios de risco da variável teor de enxofre acumulado na parte aérea da gramínea padronizada de L. multiflorum na estação amostral de Esteio, RS.

A partir dos resultados da variável Unidade Tóxica de Exposição (UTE) (Figura 3) verifica-se que os valores se situaram, principalmente, na classe de risco alta com UTE média de 145 UTE. O intervalo de concentração pico as quais as plantas poderiam ser submetidas variava de 1 hora a 720 horas (aproximadamente 30 dias de monitoramento). O tempo máximo de exposição à concentração pico foi de 265 horas, ocorrido em outubro de 2007. Estudos comprovam que há efeito na redução de crescimento de L. multiflorum, quando estes foram submetidos a concentrações controladas de $50 \mu \mathrm{g} . \mathrm{m}^{-3} \mathrm{de} \mathrm{SO}_{2}$ por três semanas (aproximadamente 504 horas) (Crittenden \& Read, 1979), bem como efeito sobre bioindicadores que apresentaram unidades tóxicas maiores do que a unidade (Tarazona, 2000). Os dados de indício de risco para o enxofre acumulado na parte aérea do bioindicador L. multiflorum (Figura 4), demonstraram equilíbrio com o percentual de risco obtido pela UTE (Figura 3) a qual considera o tempo de exposição da vegetação a concentração pico.

A integração das variáveis explicativas e resposta, obtidas pela análise estatística de correspondência (Figura 5), proporcionou o agrupamento destas em três diferentes classes de risco, quais sejam: grupo com indício de risco baixo, médio e alto (Souza, 2005; Niemeyer, 2007). Observa-se que houve correspondência entre as variáveis explicativas (UPT e UTE) e a variável resposta (enxofre foliar). Verifica-se que 
no grupo formado por indício de risco alto, os episódios que apresentaram maiores concentrações de $\mathrm{SO} 2$ no ar, ou seja, concentrações superiores a $80 \mu \mathrm{g} . \mathrm{m}-3$ apresentaram valores de UPT e UTE enquadrados como de classe de risco 3 (risco alto). Neste agrupamento também foi verificado que a variável resposta, teor de enxofre foliar, se enquadrou na classe de risco 3 (risco alto), pois o percentual de enxofre acumulado na parte aérea dos bioindicadores, nestas condições, foi superior a 0,490 , valor utilizado para intervalo de corte da classificação do risco baseada nas classes de valores-limites propostas por Klumpp et al. (2001). A Figura 5 apresenta, ainda, grupos formados para as classes de risco baixo, categorizada por valores inferiores à classe de risco 1 e grupo de classe de risco médio, categorizada por valores intermediários entre as classes de risco baixo e alto. A formação de grupos distintos proporcionou a validação do modelo proposto.

\section{REFERÊNCIAS}

ARIAS, A. R. L., BUSS, D. F., ALBUQUERQUE, C., INÁCIO, A. F., FREIRE, M. M., EGLER, M., MUGNAI, R. \& BAPTISTA, D. F., 2007, Utilização de bioindicadores na avaliação de impacto e no monitoramento da contaminação de rios e córregos por agrotóxicos. Ciênc. saúde colet., 12: 61-72. doi: 10.1590/S141381232007000100011.

ASHENDEN, T. W. \& WILliAMS, I. A. D., 1980, Growth reductions in Lolium multiflorum LAM. and Phleum pratense L. as a result of $\mathrm{SO}_{2}$ and $\mathrm{NO}_{2}$ pollution. Environ. Pollut., 21: 131139. doi: 10.1016/0143-1471(80)90041-0.

CONAMA, Conselho Nacional do Meio Ambiente, 1990, Resolução CONAMA 003 de 28 jun. 1990. Diário Oficial da União, Brasília, DF, 22 de agosto 1990.

CRITTENDEN, P. D. \& READ, D. J., 1979, The effects of air pollution on plant growth with special reference to sulfhur dioxide. III Groth studies with Lolium multiflorum LAM. And Dactylis glonerata L. New Phytol., 83:645-651. doi: 10.1111/ j.1469-8137.1979.tb02296.x.

DIVAM., A. M. Jr \& FLORES, F. E. V., 1994, Uso do Lolium multiflorum lam. (gramínease) como bioindicador de enxofre a partir das emissões de $\mathrm{SO}_{2}$ atmosférico proveniente de uma refinaria de petróleo. Dissertação de mestrado, Instituto de Biociências, Universidade Federal do Rio Grande do Sul, Brasil.

FROEHMER, S. \& Martins, R. F, 2008, Avaliação do destino e bioacumulação de benzo(A) pireno através de simulação computacional. Quim. Nova, 31: 1089-1093. doi:10.1590/S010040422008000500028 .
JUNGER, W. L., De LEON, A. P., AZEVEDO, G. \& MENDONÇA, S., 2005, Associação entre mortandade diária por câncer de pulmão e poluição do ar no município do Rio de Janeiro. Rev. bras. cancerol., 51: 111-115.

KLUMPP, A., ANSEL, W., KLUMPP, G. \& F, A., 2001, Um novo conceito de monitoramento e comunicação ambiental: a rede européia para a avaliação da qualidade do ar usando plantas bioindicadoras (EuroBionet). Rev. bras. Bot. , 24: 511-518. doi: 10.1590/S0100-84042001000500005.

LECO, 1994, Manual de Instruções do Analisador de Enxofre LECO SC-132 MODELO 781-400. FORM NO. 200-170.

MINES, O. R. Jr. \& LACKEY, L. W., 2009, Introduction to Environmental Engineering. Prentice Hall, Saddle River, New Jersey, USA, 364p.

NAKAGOME, F. K., NOLDIN, J. A. \& RESGALLA JÚNIOR, C., 2006, Toxicidade aguda e análise de risco de herbicidas e inseticidas utilizados na lavoura de arroz irrigado sobre o cladócero Daphnia magna. Pesticidas: $r$. ecotox. meio ambiente, 16: 93-100.

NIEMEYER, J. C., da Silva, E. M., \& SOUZA, J. P., 2007, Desenvolvimento de um esquema para avaliação de risco ecológico em ambientes tropicais: estudo de caso da contaminação por metais em Santo Amaro de Purificação, Bahia, Brasil. J. Braz. Soc. Ecotoxicol., 3: 263-267.

PARKHURST, B. R., WARREN-HICKS, W., CARDWELL, R. D., VOLOSIN, J., ETCHISON, T., BUTCHER, J. B. \& COVINGTON, S. M., 1996, Methodology for Aquatic Ecological Risk Assessment Contract No. RP 91-AER-1, Water Environment Research Foundation, Alexandria, VA (in press).

PEDROZO, M. F. M., 2002, Ecotoxicologia e avaliação de risco do petróleo. Centro de Recursos Ambientais, Salvador, 229p.

SESTÁK, Z., 1971, Determination of clorophylls A and B. In: Plant Phptosysthethic Production: Manual of Methods.

SOUZA, J. P., 2005, Avaliação retrospectiva do risco ambiental: esquema de avaliação de risco para solos contaminados. In: I. Abrantes \& S. Santos (eds), Manual Prático para a Gestão Ambiental. Verlag Dashofer, Lisboa.

TARAZONA, J. V., FRESNO, A., AYCARD, S., RAMOS, C., VEGA, M. M. \& CARBONELL, G., 2000, Assessing the potencial hazard of chemical substances for the terrestrial environment. Development of hazard classification criteria and quantitative environmental indicators. Sci. Total Environ., 247: 151-164.

UNITED STATES ENVIRONMENTAL PROTECTION AGENCY (USEPA), 1997, Priorities for ecological protection: an initial list and discussion document for EPA. Washington, DC: Office of Research and Development. EPA/600/S-97/002.

UNITED STATES ENVIRONMENTAL PROTECTION AGENCY (USEPA), 1989, Risk assessment guidelines for superfund. Human Health Evaluation Manual Part A. Washington, DC: Office of Research and Development. EPA/540/1-89/002. 